Apidologie, 1970, 1 (1), 55-71.

\title{
BEDEUTUNG DER TRACHEENHÜLLE UND DER ANHANGSDRÜSE DER SPERMATHEKA FƯR DIE BEFRUCHTUNGSFÄHIGKEIT DER SPERMATOZOEN IN DER BIENENKÖNIGIN (APIS MELLIFICA L.) ${ }^{1}$
}

\author{
Rôle de l'enveloppe trachéenne et de la glande de la spermathèque \\ dans le pouvoir de fécondation des spermatozoïdes \\ chez la reine d'abeille (Apis mellifica L.)
}

\section{Gudrun KOENIGER}

Institut für Bienenkunde der Universität, Frankfurt am Main, Oberursel

\begin{abstract}
SUMMARY
IMPORTANCE OF THE TRACHEAL ENVELOPE AND OF THE SPERMATHECAL GLAND IN THE FERTILISING CAPACITY OF SPERMATOZOA ON THE QUEEN-BEE ("APIS MELLIFICA L. ")
\end{abstract}

\begin{abstract}
A technique was developed enabling us to remove the spermathecal gland or the tracheal net surrounding the spermatheca in the living queenbee. By removing only one branch of the spermathecal gland in mated queens the fertilization of the eggs was not inhibited. If both branches were removed the mated queen, at the latest after a fortnight, laid non fertilized eggs, only. Generally, the motility of the spermatozoa in the spermatheca was not diminished. In spite of this fact these spermatozoa were not able to get into the spermatheca after having been taken from the operated, drone laying queen and inseminated into a young, intact queen. By removing $50-70 \%$ of the tracheal net in mated queens the spermatozoa were injured in such a manner that, after about three weeks, they grew motionless. After removal of both gland branches the pressure in the spermatheca was lessened, whereas the removal of one branch only was of no visible effect.
\end{abstract}

1. Mit Unterstützung durch Mittel der Deutschen Forschungsgemeinschaft, die Herrn Prof. RUTTNER aur Verfügung gestelit wurden. 


\section{ZUSAMMENFASSUNG}

Es wurde eine Technik entwickelt, die die operative Entfernung der Spermathekaldrüse oder des die Spermatheka umgebenden Tracheennetzes bei der lebenden Bienenkönigin ermöglichte. Durch die Entfernung nur eines Astes der Spermathekaldruse bei begatteten Königinnen wurde die Befruchtung der Eier nicht verhindert. Die Entfernung beider Äste bei begatteten Königinnen hatte jedoch zur Folge, daß nach spätestens 14 Tagen nur noch unbefruchtete Eier abgelegt wurden. In den meisten Fällen war die Motilität der in der Spermatheka gelagerten Spermatozoen nicht herabgesetzt. Trotzdem waren diese Spermatozoen, nachdem sie aus den Spermatheken der operierten, drohnenbrütigen Königinnen gewonnen und in junge, intakte Königinnen inseminiert waren, nicht mehr in der Lage, in die Spermatheka zu gelangen. Durch die Entfernung von 50-70\% des Tracheennetzes bei begatteten Königinnen wurden die Spermatozoen in der Spermatheka so geschädigt, da B sie nach etwa 3 Wochen bewegungslos wurden. Nach der Entfernung beider Drüsenäste verminderte sich der Druck in der Spermatheka, während sich die Entfernung nur eines Astes nicht sichtbar auswirkte.

\section{I. - EINLEITUNG}

Die Königin der Honigbienen nimmt auf ihrem Hochzeitsflug, der nur in den ersten Lebenswochen stattfindet, Spermatozoen in die Ovidukte (Eileiter) auf. Ein Teil von ihnen, etwa 6 Millionen, gelangt danach in ein besonderes Organ, die Spermatheka (Samenblase), in der sie für die gesamte Lebenszeit der Königin (2-6 Jahre) bei einer Temperatur von $20^{\circ}-35^{\circ} \mathrm{C}$ lebend gespeichert werden können. Die Königin kann solange befruchtete Eier ablegen, als sich in der Spermatheka ein Vorrat an funktionstüchtigen Spermatozoen befindet.

Die Spermatheka, eine kugelige Chitinblase von etwa $0,8 \mathrm{cmm}$ Volumen, ist von einem einschichtigen Epithel und einem außerordentlich dichten Tracheennetz umhüllt. Über dem Tracheennetz liegt eine zweïstige Drüse, deren Ausführgang zwischen der Bresslau'schen Samenpumpe und der Spermatheka in den Spermadukt (Samenblasengang) mündet (Abb. 1). [LaIdLAw, 1944; SNoDGRASS, 1956; RutTner, 1961].

Bisher blieb die Frage ungeklärt, auf welche Weise, die Spermatozoen in der Königin so lange befruchtungsfähig bleiben und wie die Befruchtung der Eier gesteuert wird, da fast nur Versuche mit Sperma "in vitro " oder histologische Untersuchungen durchgeführt wurden. Man hat eine Anzahl von Hypothesen vorgeschlagen, bei denen besonders der Spermathekaldrüse die verschiedensten Funktionen zugeschrieben wurden.

So wurde z.B. die Ansicht geäußert, daß das Drüsensekret Nahrungsstoffe enthalten könnte, die den Spermien während der Speicherung einen Stoffwechsel ermöglichen (RoTHSCHILD, 1955; LENSKY, 1968), wobei das Tracheennetz für den Gasaustausch sorgt.

Im allgemeinen wird jedoch angenommen, daß die Spermien in der Spermatheka inaktiviert werden, soda $\beta$ sie keinen oder nur einen geringfügigen Stoffwechsel haben (Flanders, 1950; Lensky, 1967; Taber u. Blum, 1960). In diesem Fall müßte die Chitinmembran der Spermatheka relativ undurchlässig sein, was aus der Tatsache geschlossen wurde, daß die Spermatozoen auch in verwesenden Königinnen beweglich und befruchtungsfähig bleiben (TABER und BluM, 1960). 


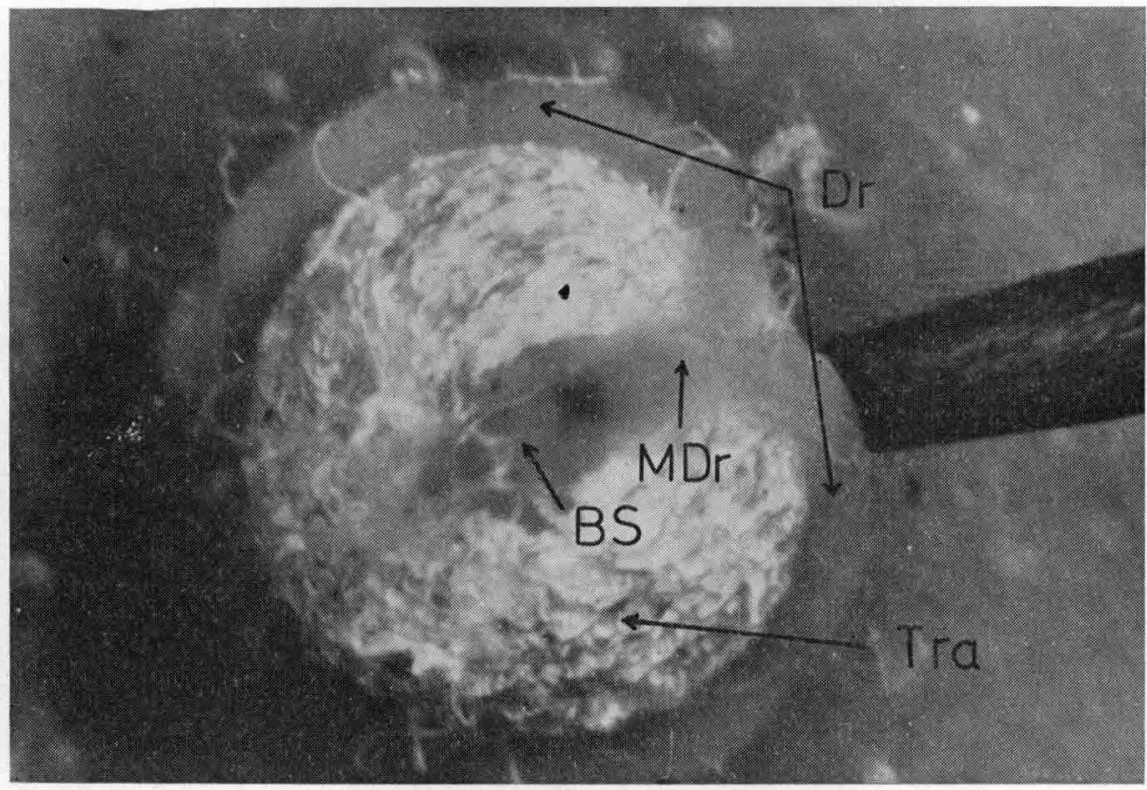

Aвв. 1. - Spermatheka mit Tacheennetz und Spermathekaldrüse

B.S. $=$ Bresslau'sche Samenpumpe, Dr. = Spermathekaldrüse (angefärbt), Tra. = Tracheennetz, M. Dr. = Mündung der Drüse in den Ductus spermaticus (Samengang).

Fig. 1. - Spermathèque avec réseau trachéen et glande de la spermathèque

B.S. $=$ Pompe séminale de Bresslau, Dr. = glande de la spermathèque (colorée), Tra. = réseau trachéen, M. Dr. = embouchure de la glande dans le Ductus spermaticus (trajet du sperme).

Bei der Befruchtung der Eier soll die Spermathekaldrüse ebenfalls eine Rolle spielen. Nach Bresslau (1905) schiebt das Drüsensekret die Spermatozoen in den Ovidukt - und zwar soll der Transport folgendermaßen vor sich gehen : Der Spermathekalgang wird durch die Bresslau'sche Samenpumpe erweitert, wodurch zunächst Sperma aus der Spermatheka angesaugt wird, das beim Verschließen des Ganges ein Stück zum Ovidukt transportiert wird. Danach wird durch denselben Mechanismus mehrmals Drüsensekret angesaugt und in den Spermathekalgang gepreßt, sodaß die Spermatozoen allmählich in den Ovidukt geschoben werden.

Nach Flanders (1946 und 1950) reguliert die Drüse die Befruchtung der Eier : Durch die Ausscheidung ihres — wie vermutet - alkalischen Sekrets entsteht ein erhöhter Druck, wodurch die S-förmige Krümmung des Spermadukts verstärkt wird, sodaß dieser verschlossen wird. Gleichzeitig soll das Sekret durch seine Alkalität die Spermien aktivieren, die während ihrer Speicherung in der Spermatheka durch die Ansäuerung des Milieus mit dem von ihnen selbst ausgeschiedenen $\mathrm{CO}_{2}$ in ein Ruhestadium versetzt werden. Jedes $\mathrm{Ei}$, das an der Mündung des Spermadukts vorbeigleitet, veranlaßt ein kurzfristiges Öffnen der Samenpumpe, und durch den herrschenden Druck gelangt eine " winzige Menge " Sperma in den Ovidukt. Bei der Ablage von Drohneneiern scheidet die Drüse kein Sekret ab, sodaß die Spermien bewegungstos bleiben. 
Aus Lenskys Untersuchungen (1967) geht jedoch hervor, daß die Spermatozoen in der Spermatheka bei einem $\mathrm{pH}-\mathrm{Wert}$ von 9 gespeichert werden, und daß der pH-Wert der Drüse 7 beträgt. Außerdem werden sie durch jegliche Verdünnung, unabhängig vom $\mathrm{pH}$-Wert des Verdünnungsmittels, aktiviert. Damit trifft die Grundannahme für die Hypothese von Flanders nicht zu. Aber auch Lensky (1967) vertritt die Ansicht, daß die Spermatozoen durch das Drüsensekret aus einem Ruhestadium aktiviert werden.

Wie diese Zusammenstellung zeigt, reichen die vorliegenden experimentellen Daten in keiner Weise zur Entwicklung begründeter Vorstellungen über die Funktionsweise der Spermatheka aus. In der folgenden Arbeit wurde die genaue Lebensdauer der Spermatozoen in toten Königinnen untersucht. Ferner wurde eine Methodik zur operativen Entfernung der Spermathekaldrüse und des Tracheennetzes bei der lebenden Königin entwickelt; auf diese Weise kann die Bedeutung dieser Organe für die Spermatozoen untersucht werden1.

\section{I. - VERHALTEN DER SPERMATOZOEN IN TOTEN KÖNIGINNEN}

Begatteten Königinnen wurden die Abdomina abgeschnitten und bei Zimmertemperatur aufbewahrt. Da die Hämolymphe an der Schnittstelle eine Kruste bildete, trockneten die isolierten Abdomina nicht aus. In verschiedenen Zeitabständen wurde die Motilität der Spermatozoen in Insekten-Ringerlösung geprüft. Sie wurden etwa 60 Stunden nach der Abtrennung der Abdomina unbeweglich.

TABeLLE 1

TABLeAU 1

$\begin{gathered}\text { Std. nach Abtrennung } \\ \text { d. Abdomen } \\ \begin{array}{c}\text { Nbre heurcs après lá résection } \\ \text { de l'abdomen }\end{array}\end{gathered}$
$\cdots$

Stufe 5 bedeutet $80-100 \%$ und 0 keine Motilität

Degré 5 signifie 80 a $100 \%$ el 0 absence de motilité

1. Herrn Prof. Dr. F. Rurrner danke ich für die Anregung zu dieser Arbeit und für die großzügige Unterstützung bei ihrer Durchführung.

Außerdem danke ich dem Beekeeping Department des Ministry of Agriculture (Israel) für einen Arbeitsplatz während des Winters 1966/1967. 
Die Spermien von 3 Abdomina, die sich etwa 20 Stunden in einem Raum befanden, in dem ein Insektizid (Paral, Fa. Thompson-Werke GmbH) gesprüht worden war, waren bereits nach 40,42 und 47 Stunden bewegungslos. Höhere Temperaturen verkürzen ebenfalls die Dauer der Motilität. Spermien in physiologischer Kochsalzlösung oder in flüssigem Paraffin bleiben dagegen 3-5 Tage beweglich. [Spermien vermischt mit Spermaflüssigkeit (Ejakulat) bleiben in zugeschmolzenen Glaskapillaren bei Zimmertemperatur bis zu 68 Tagen befruchtungsfähig (TABER, 1960)].

So ist die kurze Dauer der Motilität wahrscheinlich auf das Eindringen von Zersetzungsprodukten der Abdomina in den Spermathekałraum zurückzuführen; besonders bei Berücksichtigung der Tatsache, daß das Insektizid die Motilitätsperiode noch mehr verkürzte.

\section{III. - OPERATIVE EINGRIFFE AN DER SPERMATHEKA LEBENDER KÖNIGINNEN}

\section{A. - Material und Methode}

$\mathrm{Zu}$ den Versuchen wurden begattete, normal legende Königinnen der Rassen Apis mellifica carnica, ligustica und lamarckii (fasciata) benutzt. Sie wurden in kleinen Beobachtungskästen (Einwabenkästchen) gehalten, soda $\beta$ eine ständige Kontrolle der Königin und ihrer Eilegetätigkeit möglich war.

Der operative Eingriff wurde von der Dorsalseite aus vorgenommen, da der über der Spermatheka liegende Darm nicht wie der mediane Ovidukt am Außenskelett durch Muskeln befestigt ist und deshalb leicht verschoben werden kann. Außerdem liegen dorsal der Spermatheka keine größeren Nerven. Es wurde bei 16- und 25-facher Vergrößerung mit möglichst geringer Lichtstärke gearbeitet, um eine Verbrennung oder Austrocknung der Organe zu vermeiden.

Die Königin wurde in einem Besamungsapparat (nach MACKENSEN-RoBERTs) fixiert, der nur durch einen kleinen Holzpflock, auf dem das Abdomen der Königin lag, ergänzt wurde, und mit $\mathrm{CO}_{2}$ betäubt (Abb. 2). Der $\mathrm{CO}_{2}$ - Strom mußte so schwach eingestellt werden, da $\mathrm{B}$ die Tracheensäcke nicht gebläht wurden. Danach wurde das letzte Tergit mit einer stumpfen Pinzette, die an einem Stativ befestigt war, oberhalb des Stachelapparates gefaßt und das Abdomen gestreckt. Zur Desinfektion wurde das Abdomen mit $96 \%$ Alkohol abgerieben. Nach vollständiger Verdunstung des Alkohols wurde mit einem Rasierklingensplitter, der mit einem Antibioticum (Supracillin) versehen war, ein Fenster von max. $2 \times 2 \mathrm{~mm}$ in das vorletzte Tergit links vom Rückengefä $B$ geschnitten. Nach Untersuchungen von Fyg (1943) dürfen dabei die Intersegmentalmembranen nicht verletzt werden. Deshalb wurde der erste Schnitt quer zur Längsachse der Königin geführt, da hierbei die Intersegmentalmembran nicht gedehnt wird. Bei den beiden Längsschnitten neben dem Rückengefäß und an der Seite, die bis zur Antecosta geführt wurden, konnte das Tergit bereits mit einer Pinzette gehalten werden. Nach Einsatz des ersten Hakens in die Wunde (Abb. 3) wurde die Pinzette entfernt, sodaß das Abdomen in die Normallage zurïckkehrte. Das gelöste Chitinstück wurde an der Antecosta umgeklappt und mit einem zweiten Haken fixiert. Darauf wurde das Abdomen durch leichtes Senken der Haken flachgedrückt. Bei einer bestimmten Druckrichtung, die durch Drehung der Königin um ihre Längsachse variiert werden kann, erscheint die Spermatheka in der Öffnung (Abb. 4). Nach weiterer Freipräparierung der Spermatheka wurde entweder die Tracheenhülle von der Dorsalseite aus zu 50.70\% entfernt (Abb. 5 und 6) oder die Spermathekaldrüse wurde oberhalb ihrer Einmündung in den Spermadukt abgetrennt und herausgenommen (Abb. 7). Nach Beendigung dieser Eingriffe wurden die Haken aus der Wunde gehoben und der Chitinlappen wieder zurück geklappt ( $\mathrm{Abb}$. 8). An den Schnittstellen verkrustete nach einiger Zeit etwas Hämolymphe und bildete einen dauerhaften Wundverschiuß. 

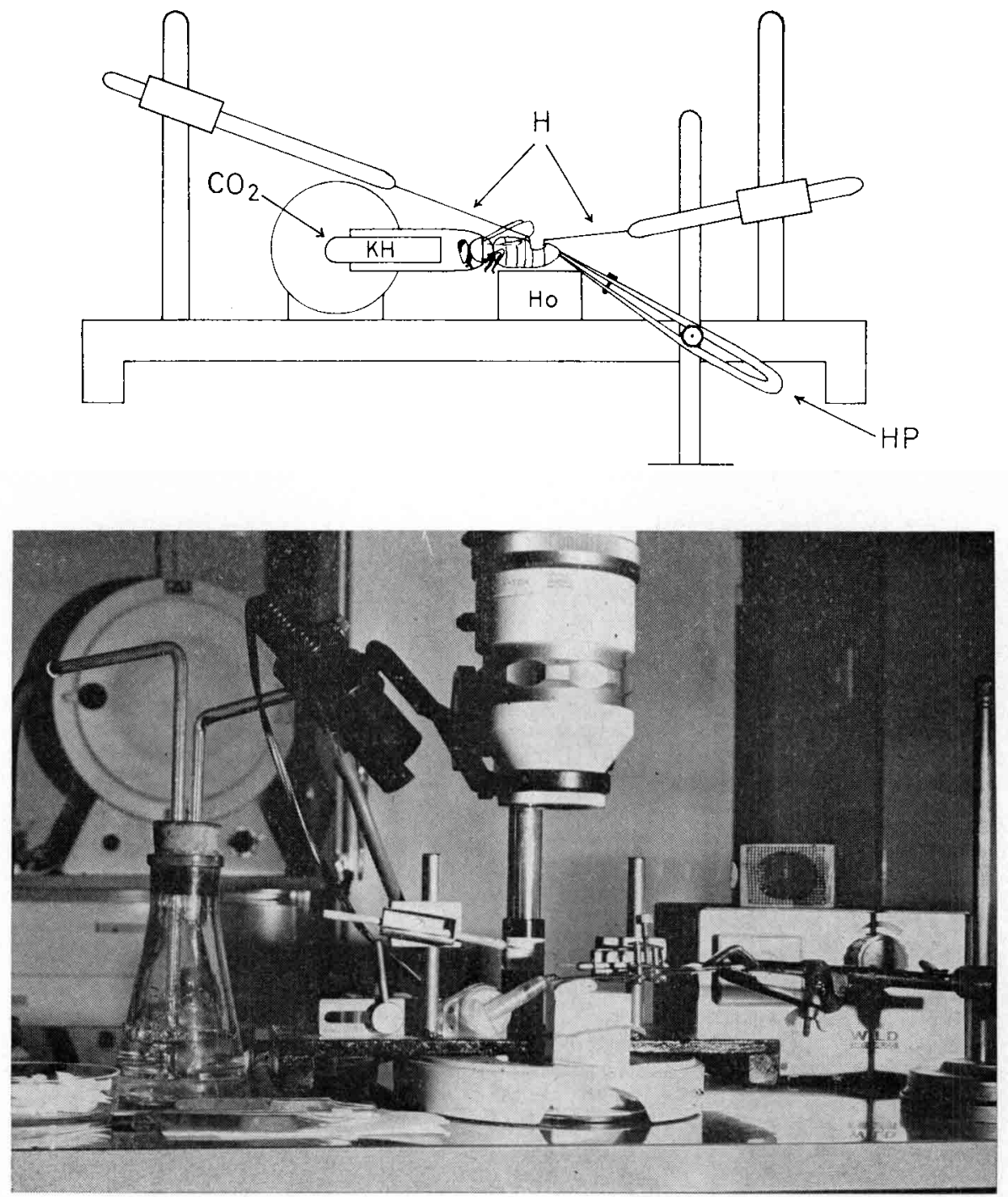

ABB. 2a. - Operationsapparatur. Schema

$\mathrm{CO}_{2}=\mathrm{CO}_{2} \cdot$ Strom zur Narkose, $\mathrm{H} .=$ Haken zur Fixierung des Operationsfensters, Ho. $=$ Holzblock zur Unterstützung des Abdomens, KH. $=$ Königinnenhalter, H.P. = Haltepinzette mit Schraubverschluß zur Streckung des Abdomens.

ABB. 2b. - Foto der Operationsapparatur mit Stereomikroskop (Wild), Besamungsapparat (MACKENSEN-ROBERTS) und Kontrollfasche für den $\mathrm{CO}_{2}$-Strom

Fig. 2a. - Appareil opératoire. Schêma

$\mathrm{CO}_{3}=$ courant de $\mathrm{CO}_{2}$ pour l'anesthésie, $\mathrm{H} .=$ crochet servant à fixer la fenêtre de chitine, HO. = bloc de bois servant à soutenir l'abdomen, KH. = logement de la reine, H.P. = pincettes avec vis de fermeture pour étirer l'abdomen.

Fic. 2b. - Photo d'appareil opératoire avec stéréomicroscope (Wild)

appareil d insémination (MACKENSEN-ROBERTs) et flacon de contrôle pour le courant de $\mathrm{CO}_{2}$ 


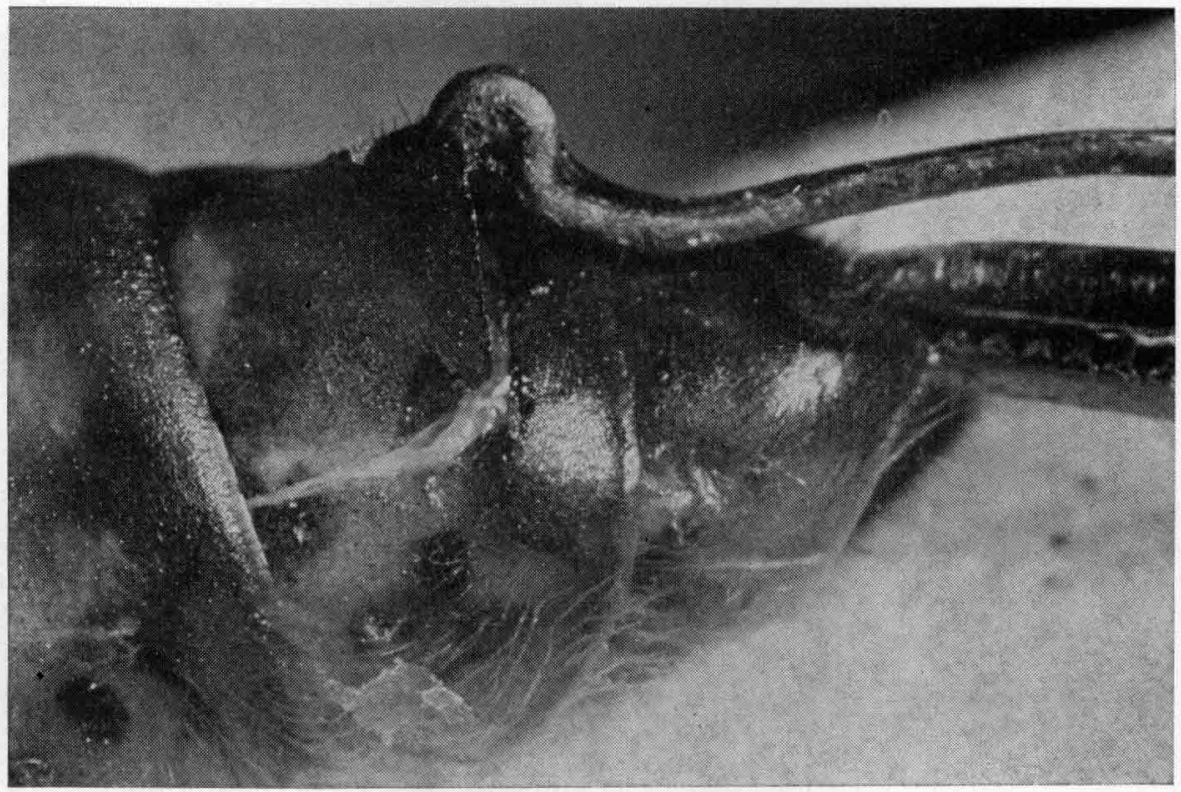

Авв. 3. - Nach_der Schnittfïhrung wurde der erste Haǩen_eingesetzt FIG. 3. - Après l'incision le premier crochet est mis en place

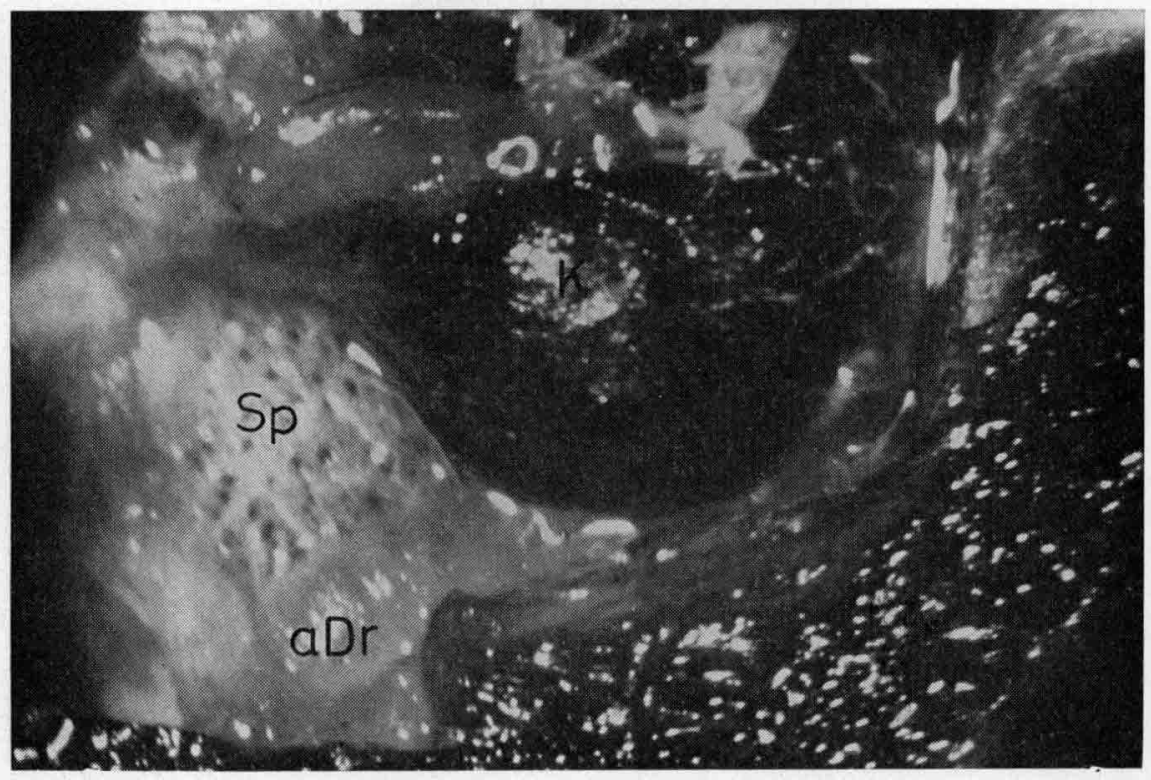

AвB. 4. - Das Chitinstitck vurde hochgeklappt und das Abdomen flachgedrückt a. Dr. = alkalische Drüse, K. $=$ Kotblase, Sp. $\Rightarrow$ Spermatheka.

Frg. 4. - Le morceau de chitine a été relevé et l'abdomen aplati a. Dr. = glande alcaline, K. = ampoule rectale, Sp. = spermathèque. 


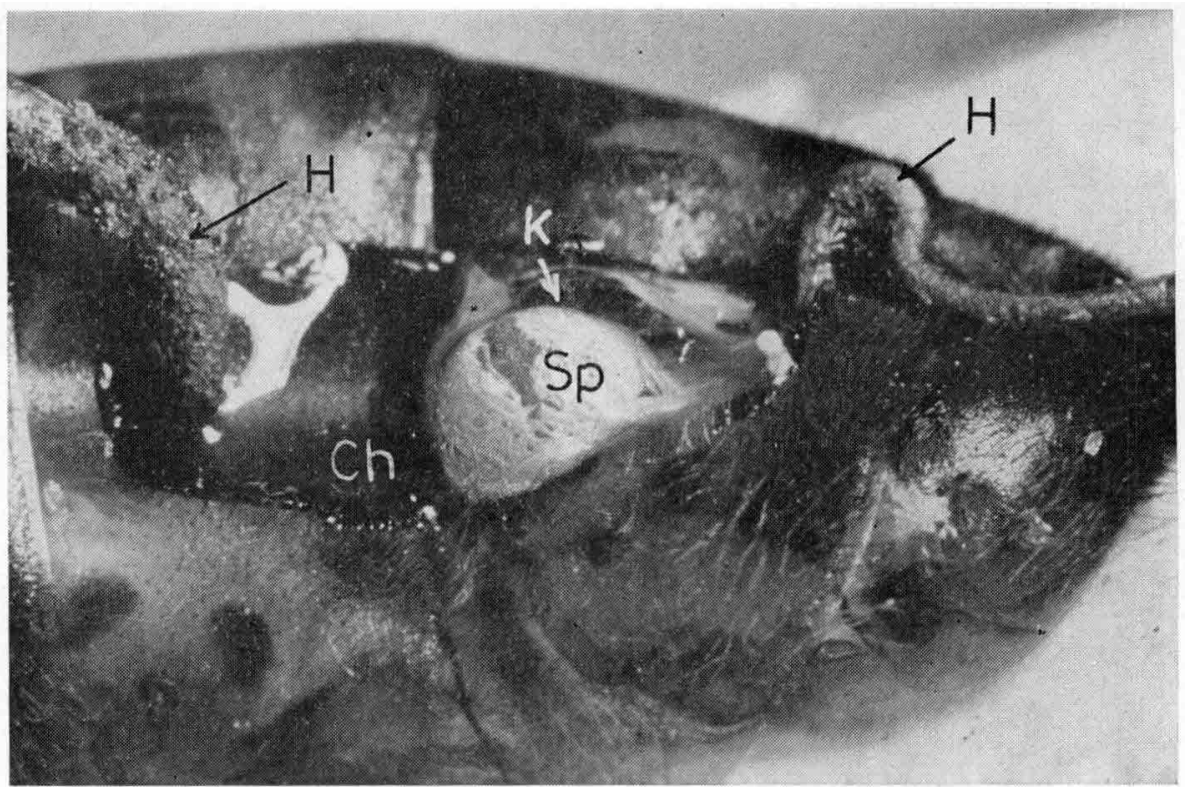

Авв. 5. - Die Spermatheha wurd̉e weiter freipräpariert und̉ mit d̉er Entf̂́ernung der Tracheenhülle begonnen

H. = Haken zur Fixierung des Operationsfensters, Ch. $=$ aufgeklapptes Chitinstück, K. = Kotblase, Sp. = Spermatheka.

FIG. 5. - La spermathèque subit ensuite une préparation

et on commence l'élimination de l'enveloppe trachéenne

$H$. = crochet servant à fixer la fenêtre de chitine, Ch. = Morceau de chitine soulevé, $K .=$ ampoule rectale, $\mathrm{Sp}$. $=$ spermathèque.

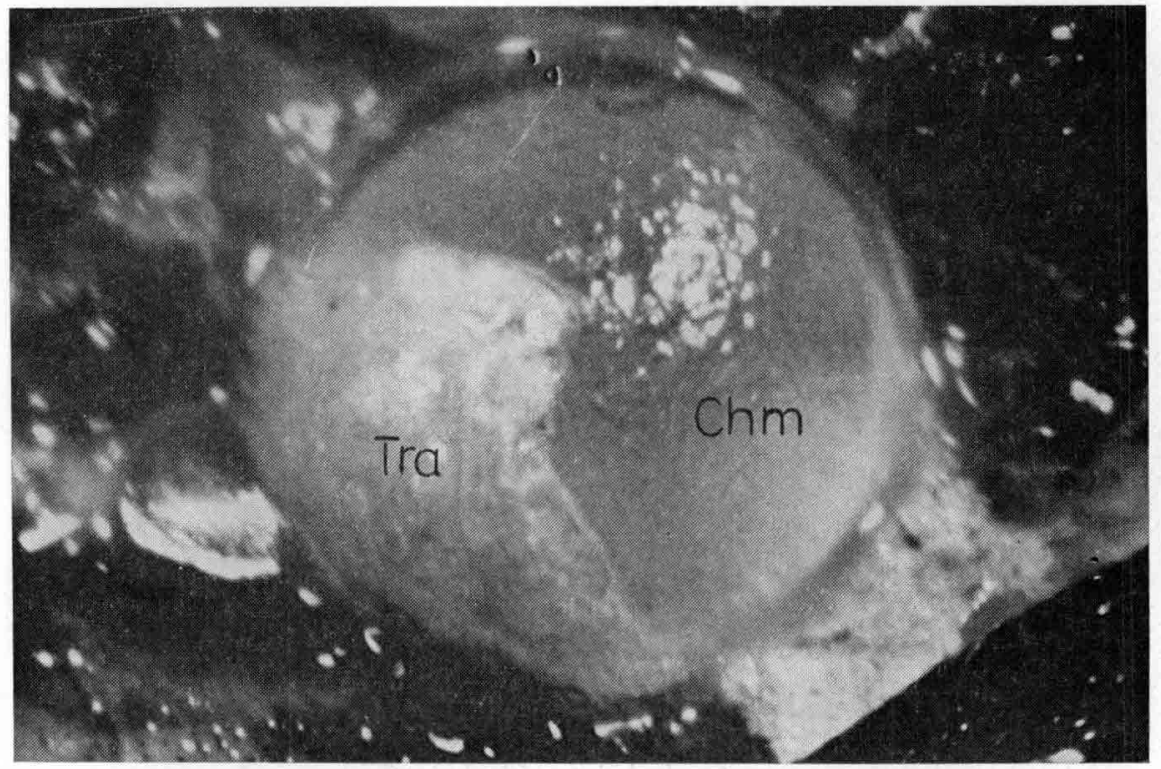

ABB. 6. - Die Tracheenhïlle wurde zu einem groß̂̉en Teil entfernt

Chm. = Chitinmembran der Spermatheka, Tra. = Rest der Tracheenhülle.

Fig. 6. - La plus grande partie de l'enveloppe trachéenne a été supprimée

Chm. = membrane chitineuse de la spermathèque, Tra. = Reste de l'enveloppe trachéenne. 


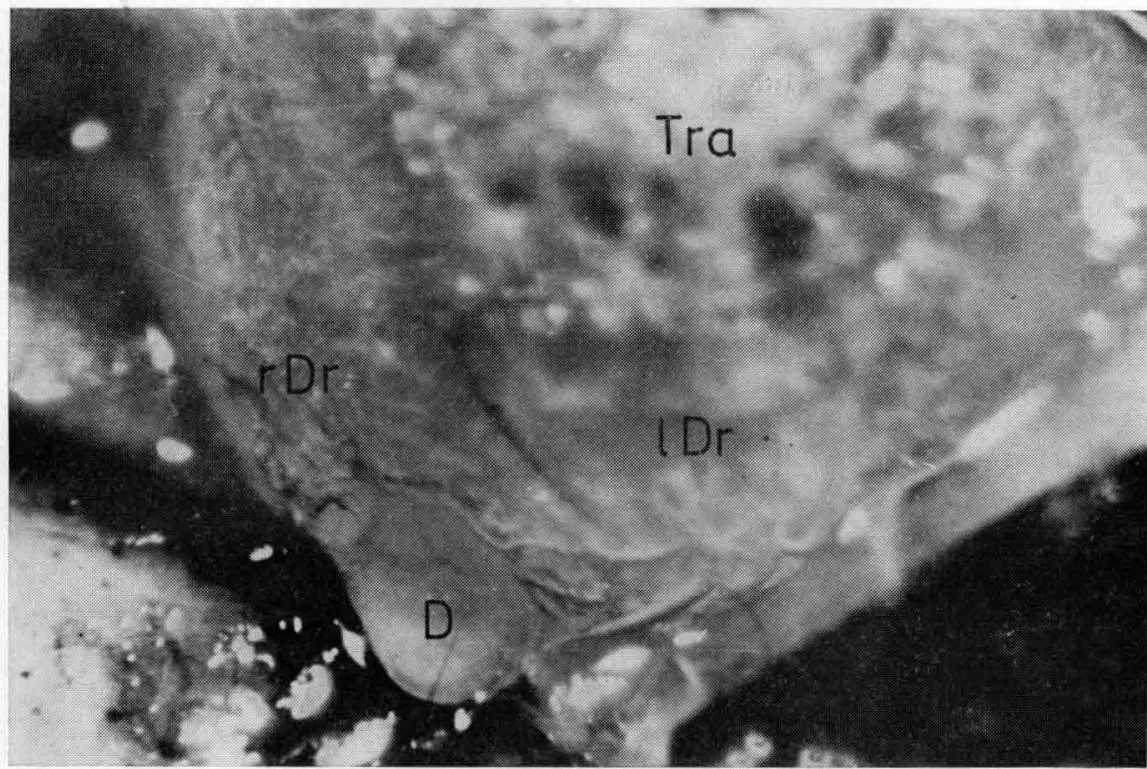

Aвв. 7. - In diesem Operationsstadium wurde die Spermathekaldrüse entfernt

D. = Ductus spermaticus, 1. Dr. = linker Ast der Spermathekaldrüse, r. Dr. $=$ rechter Ast der Spermathekaldrüse, Tra. = Tracheenhülle.

Fig. 7. - A ce stade de l'opération la glande de la spermathèque a été enlevée

D. Ductus spermaticus; 1 . Dr. = ramification gauche de la glande de la spermathèque, r. Dr. = ramification droite de la glande de la spermathèque, Tra. = enveloppe trachéenne.

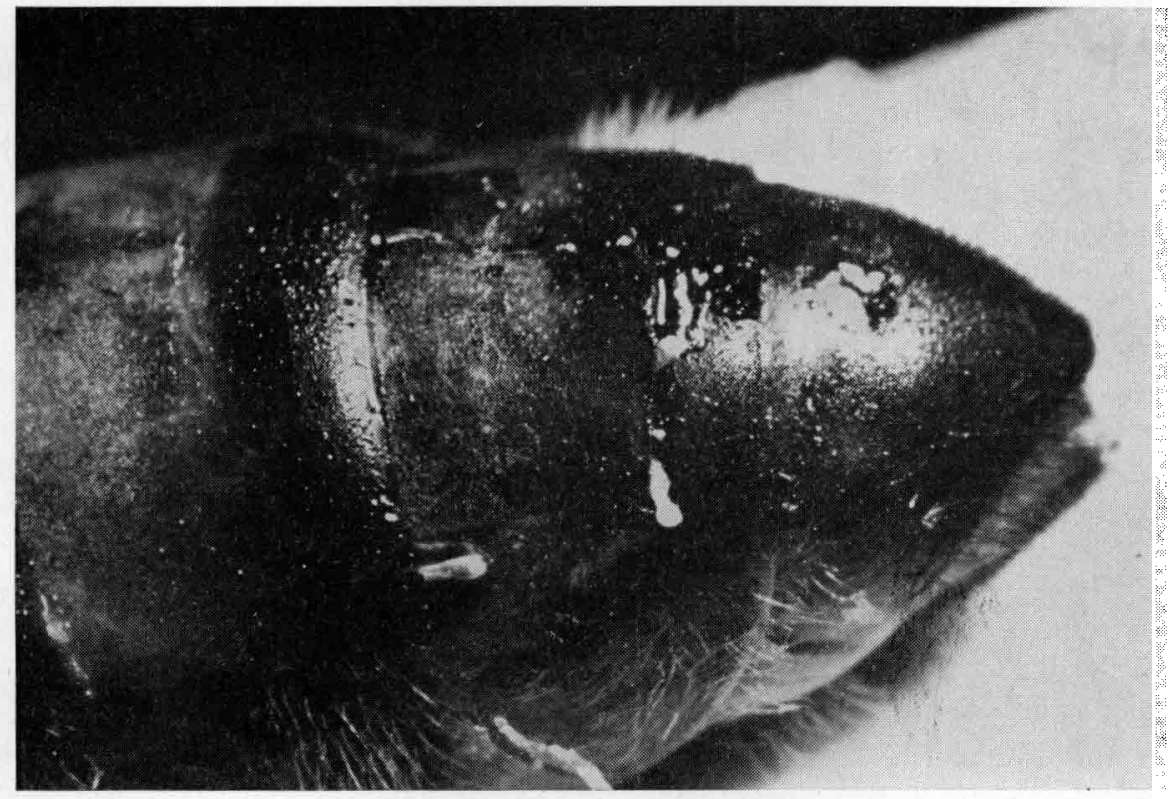

Aвв. 8. - Die Wunde wurde durch Zurückhlappen des Chitinstückes verschlossen

Fig. 8. - La blessure est refermée en rabattant le morceau de chitine 
Die Dauer der Operation betrug zwischen 10 und 20 Minuten. Sobald sich die Königinnen nach der Narkose soweit erholt hatten, da $\beta$ sie sich auf senkrechten Wänden halten konnten, wurden sie direkt ins Volk zurückgesetzt. In der Regel begannen die Bienen sofort mit der Fütterung und beleckten intensiv die Schnittstellen. Nur in zwei Fällen wurde dabei die Wunde wieder geöffnet. (Das Belecken könnte eine desinfizierende Wirkung haben, da fast alle Königinnen starben, die in Zusetzkäfigen nach der Operation dem Volk zurückgegeben wurden.)

In verschiedenen Zeitabständen nach der Operation wurden die Königinnen getötet und sorgfältig präpariert, um zu kontrollieren, ob der Eingriff gelungen war. In einem Schema wurde jeweils der Anteil der verbliebenen Drüsenreste eingetragen (Tab. 2 und 4). Die Aktivität der Spermatozoen wurde in physiologischer Kochsalzlösung oder in Insekten-Ringerlösung untersucht.

Als erfolgreiche Operation wurde gewertet, wenn die Königin nach dem Eingriff wieder in Eiablage ging. Sobald die Entwicklung der eben beschriebenen Technik genügend weit fortgeschritten war konnten etwa $60 \%$ der operierten Königinnen zur Auswertung herangezogen werden. Betrachtet man allerdings die Gesamtzahl der Königinnen, die operiert wurden, so waren nur etwa $23 \%$ der Operationen erfolgreich, da in den ersten Serien fast alle Königinnen starben, ohne wieder in Eiablage zu gehen. (Einschließlich aller Vorversuche wurden etwa 250 Königinnen operiert).

Um das Verhältnis von Arbeiterinnen zu Drohnen nach der Operation zu bestimmen, wurden die Brutwaben in einen Brutschrank gestellt, und 10-15 Tage später die Imagines und Puppen ausgezählt.

Die Zählung der Spermatozoen erfolgte nach der Methode von Mackensen-Roberts, wobei eine Zählkammer nach Fuchs-RosenTaL benutzt wurde.

\section{B. - Entfernung der Spermathekaldrüse bei begatteten Königinnen}

Zwei begatteten Königinnen (320 und 332) wurde 1 Ast der Drüse entfernt. Diese Königinnen legten während der nächsten 21 bzw. 41 Tage weiterhin reine Arbeiterinnenbrut (Tab.2). Auch zwei Königinnen, die erst nach der Entfernung eines Drüsenastes begattet wurden, erzeugten in einem Fall ausschließlich Arbeiterinnen, im anderen nur vereinzelt Drohnen. Die Spermatozoen dieser Königinnen waren bei der Kontrolle normal beweglich.

Nach Entfernung beider Drüsenäste begannen alle Königinnen im Laufe der nächsten 14. Tage unbefruchtete Eier abzulegen. Zumindest ein Teil dieser Königinnen hatte vorher (also zwischem dem 1. und 12. Tag nach der Operation) noch befruchtete Eier gelegt. Der Zeitpunkt der Ablage der ersten unbefruchteten Eier wurde bestimmt, indem vom Datum des Erscheinens der ersten hochverdeckelten Zellen 10 Tage abgezogen wurden.

Bei der Kontrolle der Königinnen stellte sich heraus, da $B$ der Druck in den Spermatheken nachgelassen hatte, soda $B$ diese schlaff waren. Die Spermien verhielten sich in physiologischer Kochsalzlösung bei 11 Königinnen "normal» (5+, s. Tab. 2); in einigen Fällen wurde die Dauer der Motilität getestet : sie betrug bis zu 5 Tagen, selbst bei Königin 17, der 77 Tage zuvor die Drüse entfernt worden war. Bei 5 Königinnen hatte die Bewegdichkeit der Spermatozoen deutlich nachgelassen, wobei eine Abhängigkeit von dem Zeitraum zwischen der Operation und der Kontrolle nicht zu bestehen scheint. Der einzige Zusammenhang, der zwischen den Werten zu sehen ist, besteht darin, daß in diesen Fällen die Spermatheka gedellt war, während sie sonst nur schlaff war. Bei Königin 105 wurde die Spermatheka während der Operation angestochen.

Bei diesem Ergebnis muß berücksichtigt werden, daß noch kein Nachweis vorliegt, ob aus dem Verhalten der Spermatozoen in physiologischer Kochsalzlösung auf ihre Befruchtungsfähigkeit geschlossen werden kann. Deshalb wurde folgender Versuch unternommen : Von vier operierten Königinnen wurden die 
ТАв. 2. - Einfluß der operativen Entfernung von der Spermathekaldrïse auf die Motilität der Spermatozoen, die Befruchtung der Eier und die Druckverhältnisse in der Spermatheka.

TaBL. 2. - Influence de l'êlimination opératoire de la glande de la spermathèque sur la motilité des spermatozoïdes, la fécondation des ceufs et les conditions de pression dans le spermathèque

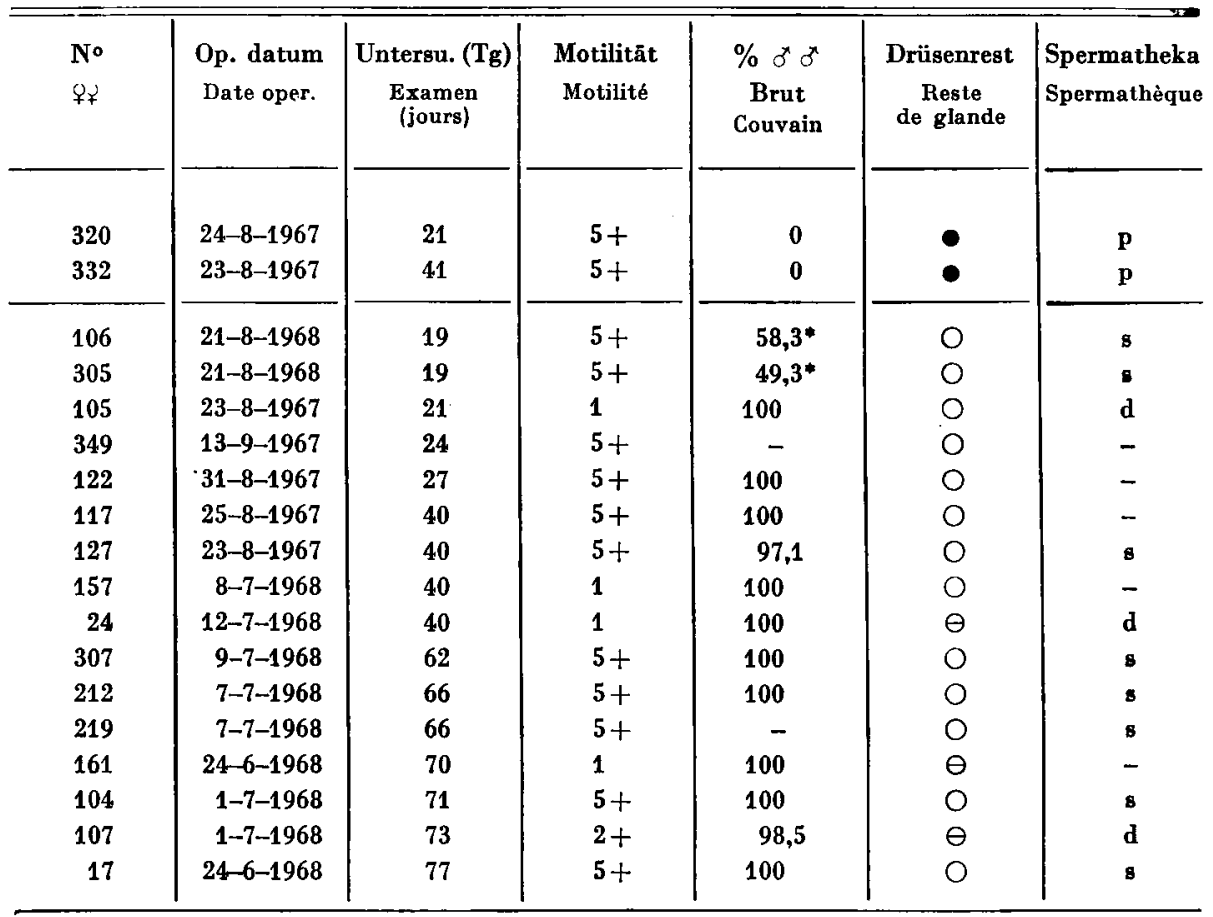

5 bedeutet $80-100 \%$ und 0 keine Motilität signifie 80-100\% et 0 aucune motilité.

+ bedeutet Anlagerung an feste Oberflächen und koordinierter Schwanzschlag signifie dépót sur les surfaces solides et battement coordonné des flagelles.

$O$ die ganze Spermathekaldrüse wurde entfernt $=$ toute la glande a été enlevée.

- ein Ast der Spermathekaldrüse wurde entfernt = seule une branche de la glande a été enlevée.

$\ominus$ die Verbindung der Drüse mit dem Ductus spermaticus wurde unterbrochen $=$ la liaison entre la glande et le Ductus spermaticus a été interrompue.

$p$ prall/turgescent.

$s$ schlaff/relaché.

d gedellt/enfoncé.

* $\Varangle \zeta$ schlüpften nur in den ersten Tagen nach der Untersuchung der $\$ \ngtr$.

¡ఢ ne sont écloses que les premiers jours après l'examen des

Spermatozoen aus den Spermatheken entnommen und jeweils eine junge, intakte Königin damit künstlich besamt ${ }^{1}$. Bei zwei Königinnen (106 und 305) war die Drüse 19 Tage vor Entnahme der Spermatozoen entfernt worden, während bei einer Königin (307) 62 Tage und bei Königin 104 seit der Entfernung der Drüse

1. Die künstlichen Besamungen wurden freundlicherweise von Herrn Prof. Dr. F. Rutrines durchgeführt. 
71 Tage vergangen waren. Alle Königinnen waren zum Zeitpunkt der Spermaentnahme bereits drohnenbrütig. Die entnommenen Spermatozoen waren ohne Ausnahme in physiologischer Kochsalzlösung normal beweglich.

Von den Spermatozoen der Königin 106 konnten etwa 2000 in der Spermatheka der inseminierten Königin nachgewiesen werden. Bei den anderen drei inseminierten Königinnen wurden vereinzelte oder gar keine Spermatozoen nachgewiesen. Eine dieser Königinnen konnte zur Eiablage gebracht werden (Drohneneier), während die anderen im Laufe von drei Wochen starben (wohl wegen der schon fortgeschrittenen Jahreszeit). Bei dem Kontrollversuch wurden Spermatozoen aus einer "normalen" Königin gewonnen und inseminiert. Es gelangten etwa $100^{\prime} 000$ in die Spermatheka. Auch konnte bei dieser Königin die Ablage von befruchteten Eiern neben nur wenigen Drohneneiern beobachtet werden (Tab. 3).

TABELLE 3

Tableau 3

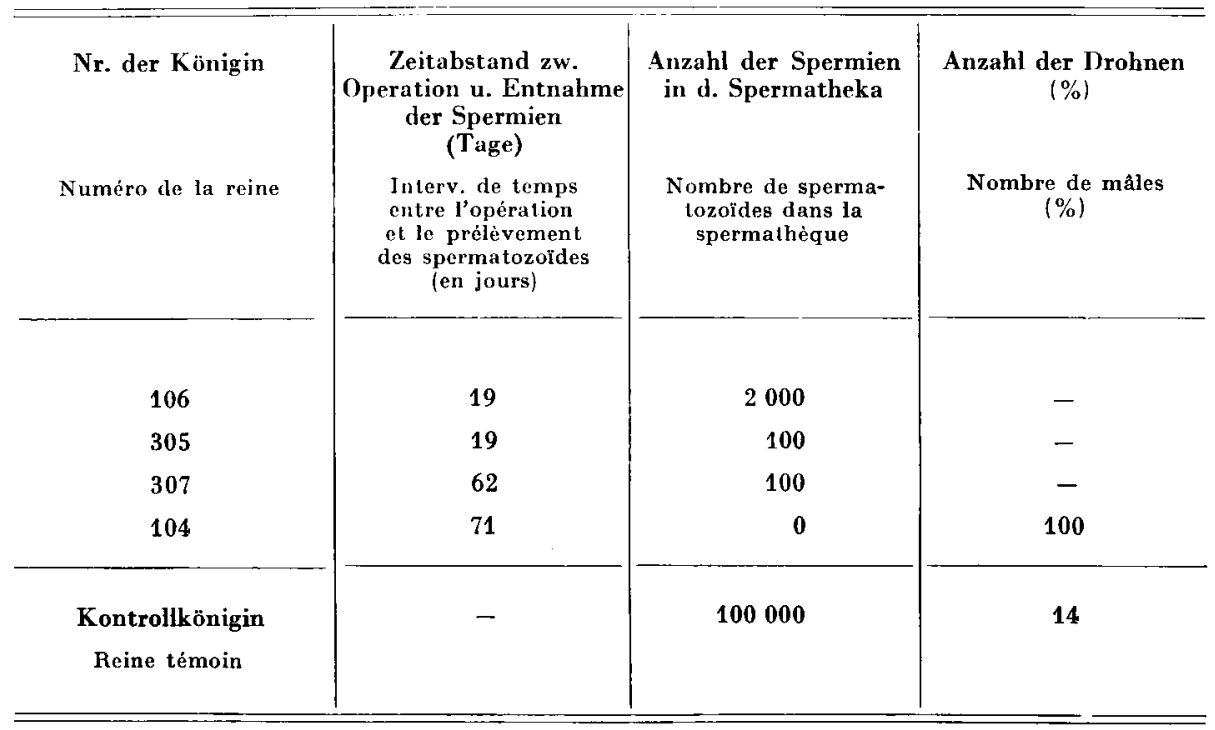

Die Spermatozoen waren also durch die Entfernung der Drüse so geschädigt, daß sie nicht mehr in der Lage waren, in die Spermatheka zu gelangen, obwohl sie sich in physiologischer Kochsalzlösung normal verhielten.

Aus dem normalen Verhalten der Spermatozoen in physiologischer Kochsalzlösung darf also nicht geschlossen werden, daß diese völlig intakt sein. Dagegen scheint es zulässig, aus verminderter Beweglichkeit auf eine Schädigung zu schließen. 


\section{C. - Entfernung des Tracheennetzes bei begatteten Königinnen}

Bei 7 begatteten Königinnen wurden 50-70\% des die Spermatheka umgebenden Tracheennetzes ohne Verletzung der Spermathekaldrüse abpräpariert (Tab. 4). Auch bei diesem Versuch legten die meisten Königinnen zunächst

Tав. 4. - Einflu $\beta$ der operativen Entfernung von 50-70\% des Tracheennetzes auf die Motilität der Spermatozoen.

TaBL. 4. - Inflaence de l'élimination opératoire de 50 à $70 \%$ du réseau trachéen sur la motilité dos spermatozoïdes.

\begin{tabular}{|c|c|c|c|c|c|}
\hline No 77 & $\begin{array}{l}\text { Op. datum } \\
\text { Date op. }\end{array}$ & $\begin{array}{l}\text { Untersuchung } \\
\quad(\mathrm{Tg}) \\
\text { Examen (jours) }\end{array}$ & $\begin{array}{l}\text { Motilität } \\
\text { Motilité }\end{array}$ & $\begin{array}{c}\% \sigma^{x} \\
\text { Brnt } \\
\text { Couvain }\end{array}$ & $\begin{array}{l}\text { Drüsenrest } \\
\text { Reste } \\
\text { de la glande }\end{array}$ \\
\hline 37 & $22-8-1967$ & 5 & $5+$ & - & - \\
\hline 206 & $20-7-1968$ & 16 & 0 & - & - \\
\hline 52 & $20-7-1968$ & 17 & 1 & $28,4^{*}$ & - \\
\hline 313 & $20-7-1968$ & 17 & $5+$ & $32,8^{*}$ & - \\
\hline 295 & $19-7-1968$ & 18 & $5+$ & $14,3^{*}$ & $\bullet$ \\
\hline 113 & $12-7-1968$ & 24 & 0 & $64,1^{*}$ & - \\
\hline 214 & $12-7-1968$ & 27 & 1 & 100 & - \\
\hline 239 & $19-7-1968$ & 30 & 0 & 100 & $\bullet$ \\
\hline 237 & $19-7-1968$ & 30 & 0 & 100 & - \\
\hline 156 & $24-8-1967$ & 40 & 0 & 100 & 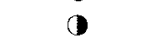 \\
\hline 59 & $6-8-1967$ & 40 & 0 & 100 & 0 \\
\hline 79 & $24-6-1968$ & 41 & 0 & 100 & 1 \\
\hline
\end{tabular}

5 bedeutet $80-100 \%$ und 0 keine Motilität signifie $80-100 \%$ et 0 aucune motilité

+ bedeutet Anlagerung an feste Oberflächen und koordinierter Schwanzschlag signifie dépòt sur les surfaces solides et battement coordonné des flagelles.

- Spermathekaldrüse blieb unverletzt = la glande de la spermathèque est restée indemne.

ein Ast der Spermathekaldrüse wurde entfernt $=$ une branche de la glande a été enlevée.

$O$ die ganze Spermathekaldrüse wurde entfernt = toute la glande a été enlevée.

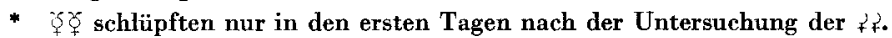
孔̧ ne sont écloses que les premiers jours après l'examen des $\Varangle$.

befruchtete Eier, wurden aber im Laufe der nächsten 14 Tage drohnenbrütig, und nach weiteren 1-2 Wochen waren in allen Fällen die Spermien unbeweglich. Bei Königin 156 und 79 wurde außerdem unabsichtlich ein Ast der Spermathekaldrüse, bei Königin 59 beide Äste entfernt. Die Königinnen wurden zu dieser Versuchsreihe gestellt, weil auf Grund der bereits beschriebenen Experimente gezeigt werden konnte, daß sich die Entfernung von nur einem Ast der Drüse überhaupt nicht, von der ganzen Drüse nur geringfügig auf die Motilität auswirkt. 
Bei Königin 313 und 295 wurde das Tracheennetz nicht entfernt, sondern lediglich gelöst. Bei der Untersuchung dieser Königinnen befand sich die Tracheenhüille seitlich zum Spermadukt verschoben, sodaB etwa $80 \%$ der Spermatheka frei war. Innerhalb von 17 bzw. 18 Tagen wirkte sich dieser Eingriff auf die Motilität der Spermien nicht aus. Allerdings wurden auch diese Königinnen drohnenbrütig.

\section{DISKUSSION}

Mit Kontrollversuchen - den Königinnen wurde nur ein Ast der Drüse entfernt - konnte gezeigt werden, daß bei der Operation keine für die Befruchtung der Eier wichtigen Organe verletzt oder in ihrer Funktion gestört wurden, da alle diese Königinnen befruchtete Eier legten. Außerdem wurden im allgemeinen nur Königinnen ausgewertet, die ihre Legetätigkeit fortsetzten. Das ist ein sehr empfindliches Maß für das "Wohlbefinden » der Königin, denn jede größere Störung führt zur Einstellung der Eiablage.

Nach Entfernung beider Drüsenäste bei begatteten Königinnen begannen diese innerhalb von 14 Tagen unbefruchtete Eier abzulegen. Die Spermatozoen waren 77 Tage nach der Operation noch normal beweglich. Trotzdem waren sie durch die Entfernung der Spermathekaldrüse so geschädigt, daß sie nach der Insemination in eine normale junge Königin nicht mehr fähig waren, in die Spermatheka zu gelangen. Da diese Schädigung der Spermatozoen nicht durch die offene Verbindung des Spermathekałraumes mit der Hämolymphe hervorgerufen werden kann, wie die Versuche an Königinnen mit nur einem Drüsenast zeigen, ist sie entweder auf das Fehlen bestimmter Stoffe des Drüsensekrets oder auf den gesunkenen Druck in der Spermatheka zurückzuführen. Auf jeden Fall scheint die Drüse durch die Abscheidung des Sekrets den Druck in der Spermatheka aufrecht zu erhalten, denn nach der Entfernung eines Drüsenastes blieb der Druck im wesentlichen erhalten.

Durch die Entfernung von 50-70\% der Tracheenhülle der Spermatheka wurden die Spermatozoen innerhalb von 3 Wochen bewegungslos. Sie scheinen also während der Speicherung auf Sauerstoffversorgung angewiesen zu sein.

Bei den Königinnen 313 und 295 wurden die Tracheenhüllen vollständig von der Sprematheka geschoben und zusammengeballt um den Spermadukt in der Königinnen belassen. Nach diesem Eingriff wurden die Königinnen zwar auch drohnenbrütig, aber die Spermien waren 17 bzw. 18 Tage später noch normal beweglich, während sie bei zwei anderen Königinnen, denen 16 und 17 Tage zuvor 50-70\% der Tracheenhülle entfernt worden war, bereits fast unbeweglich waren. Dieser Befund kann vielleicht als ein Hinweis gedeutet werden, daß der Gasaustausch nicht durch Tracheenendzellen, die direkt an der Chitenmembran anliegen, erfolgt, sondern daß eine Sauerstoffanreicherung im Perispermathekalraum vorliegt. Leider liegen bisher noch keine histologischen Untersuchungen vor. 
Durch den Nachweis eines Gasaustausches durch die Chitinmembran der Spermatheka ist die Hypothese von Flanders, daß sich die Spermatozoen durch die Ansäuerung des Milieus mit dem von ihnen ausgeschiedenen $\mathrm{CO}_{2}$ selbst inaktivieren, ebenfalls widerlegt (s. S. 2).

Die Chitinmembran ist aber wahrscheinlich nicht nur für Sauerstoff, sondern auch für größere Moleküle durchlässig, wie die kurze Lebensdauer der Spermatozoen in toten Königinnen und die Vergiftungserscheinungen durch Insektizide zeigen. Auch die Untersuchungen von Erdmans (1922) über die Durchlässigkeit der Chitinintima aus dem Enddarm von Blatta spec. - der Durchtritt von $\mathrm{H}^{+}$und $\mathrm{OH}^{-}$- Ionen ließ sich bei einer $2 \mu$ dicken Membran nach 10 Minuten kolorimetrisch nachweisen - sprechen gegen einen vollständigen Abschluß der Spermatozoen durch die Chitinmembran, die dünner als $2 \mu$ ist. Es erscheint möglich, daß das einschichtige Epithel als biologische Membran dient, denn häufig sind in den Epithelzellen von Spermatheken, die abgestorbene Spermatozoen enthalten, Ablagerungen von amyloiden Stoffen zu beobachten, die vielleicht als Ausdruck einer funktionellen Schädigung zu werten ist (Fyg, 1960).

Ob der an die Sauerstoffversorgung geknüpfte Stoffwechsel auf 'dem Abbau von spermaeigenen Reservestoffen beruht, oder ob eine Nahrungszufuhr durch die Königin erfolgt, kann nicht entschieden werden. In diesem Zusammenhang muß die Rolle der Spermathekaldrüse noch eingehender untersucht werden. Nach elektronenmikroskopischen Untersuchungen an der Spermatheka von Aedes aegypti (Clements und Potter, 1967) ist die Chitinmembran "almost certainly impermeable to nutritive materials. The only source-of nourishment for the stored sperm can be the products of the female's glandular cells, unless nutritive materials in the male's accessory gland secretions enter the spermathecae with the sperm. " (... " fast sicher für Nahrungsstoffe undurchlässig. Die einzige Nahrungsquelle für die gespeicherten Spermien können die Produkte der weiblichen Drüsenzellen sein, wenn nicht Nahrungsstoffe im Sekret der männlichen akzessorischen Drüse mit dem Sperma in die Spermatheka eindringen. ")

Nach den eigenen Ergebnissen ist es unwahrscheinlich, daß das Sekret der Drüse nur als Treibmittel oder als Aktivator dient, denn in diesen Fällen hätten die Spermatozoen nach der erneuten Insemination die Spermatheka erreichen müssen. Vielmehr ${ }^{-} \mathrm{mu}$ angenommen werden, daß das Drüsensekret einen für die Lokomotion und Befruchtungsfähigkeit der Spermatozoen essentiellen Faktor enthält, dessen Fehlen zu einer irreparablen Schädigung führt.

Auf jeden Fall eröffnet die Methode der Operation im Bienenabdomen die Möglichkeit zur Durchführung subtilerer Eingriffe, die auch in anderem Zusammenhang als dem hier erörterten Fragenkomplex von großem Interesse sein dürften. 


\section{RÉSUMÉ}

Pour connâ̂tre le rôle de la glande de la spermathèque et de l'enveloppe trachéenne (fig. 1) dans la conservation des spermatozoìdes on a procédé à une opération sur la reine vivante. Celle-ci est anesthésiée et placée dans un appareil à insémination modifié, l'abdomen étant bien étiré (fig. 2). Après désinfection de l'abdomen on découpe avec un éclat de lame de rasoir une fenêtre de $2 \times 2 \mathrm{~mm}$ maximum dans la chitine dure de l'avant dernier tergite (fig. 3). Le morceau de chitine détaché est placé vers l'avant sur l'antecosta et fixé. La spermathèque, qui doit se trouver juste au-dessous de l'ouverture, subit une préparation et, par une pression sur l'abdomen, fait légèrement saillie par l'orifice (fig. 4, 5). A ce moment on prélève une partie du réseau trachéen ou de la glande de la spermathèque (fig. 6, 7). Le morceau de chitine est ensuite remis en place (fig. 8). La blessure se referme d'elle même par l'action de l'hémolymphe.

L'opération dure entre 10 et 20 minutes. A différents intervalles de temps après l'opération, les reines sont tuées et soigneusement préparées pour vérifier si l'intervention a été efficace. L'activité des spermatozoỉdes est étudiée dans une solution physiologique de chlorure de sodium. L'opération est considérée comme réussie lorsque les reines recommencent à pondre après l'intervention. Cette technique a pu être suffisamment élaborée pour que finalement environ $60 \%$ des opérations soient réussies. La suppression totale de la glande chez 16 reines fécondes provoque la ponte de mâles au bout de 14 jours sans exception (tabl. 2). On a constaté, lors de l'examen des spermathèques, que les spermatozoìdes se comportaient en général normalement dans une solution physiologique au chlorure de sodium, même chez une reine dont la glande avait été supprimée 77 jours auparavant. Pour vérifier l'état des spermatozoīdes, on a prélevé dans la spermathèque ceux de 4 reines opérées qui pondaient des mâles et on les a introduits, par insémination, dans une jeune reine intacte. Chez 3 de ces reines presqu'aucun spermatozoïde n'a atteint la spermathèque, chez la $4^{\mathrm{e}}$ une proportion de $2 \%$ avait atteint la spermathèque au moment de l'expérience de contrôle. 4 reines témoins, auxquelles on n'avait enlevé qu'une ramification glandulaire, ont pondu des œufs fécondés (tabl. 3). Chez 12 reines fécondes on a retiré 50 et $70 \%$ de l'enveloppe trachéenne (tabl. 4). Mème ces reines se sont mises à pondre des mâles au plus tard 14 jours après l'opération. On a examiné les spermatozoïdes dans le liquide physiologique : 3 semaines environ après l'opération ils étaient immobiles.

On a pu montrer par des expériences témoins (en supprimant 1 seule ramification de la glande chez les reines) qu'aucun organe important dans la fécondation des œufs n'a été endommagé par l'opération ou gêné dans sa fonction puisque toutes ces reines pondaient des œufs fécondés.

La suppression des deux ramifications glandulaires chez des reines fécondes provoque également la ponte d'œufs non fécondés. Comme on a pu le prouver par l'insémination artificielle de reines intactes avec des spermatozoïdes de reines opérées, l'absence de la glande cause un préjudice aux spermatozoìdes. L'ouverture de l'enceinte de la spermathèque à l'hémolymphe ne peut avoir aucune influence sur les spermatozoïdes puisque, avec une seule ramification glandulaire, les reines pondent des œufs fécondés.

La suppression de 50 à $70 \%$ du réseau trachéen cause un tel préjudice aux spermatozoïdes qu'au bout de 3 semaines ils ont perdu leur motilité. Il semble donc que, dans la spermathèque, les spermatozoïdes ont besoin d'un approvisionnement en oxygène, c'est-à-dire que pendant leur conservation ils ont un métabolisme. On ne sait pas encore d'où proviennent les substances nutritives. Pour résoudre ce problème il est nécessaire d'étudier de façon plus détaillée le rôle de la glande de la spermathèque. 


\section{LITERATUR}

Blum S.M., Taber S., 1960. - Preservation of Honey Bee Semen. Science, 131, 1734-1735.

Blum S.M., Glowska Z., Taber S., 1962. - Chemistry of the Drone Honey Bee Reproductive System II. Carbohydrates in the Reproductive Organs and Semen. Ann. Entomol. Soc. Amer., $55,135-139$.

Buum S.M., TABer S., 1965. - Chemistry of the Drone Honey Bee Reproductive System III. Dehydrogenases in washed Spermatozoen. J. Insect. Physiol., 11, 1489-1501.

Bresslau E., 1905. - Der Samenblasengang der Bienenkönigin. Zool. Anz. 29, 299-325.

Clements A.N., Potter S.A., 1967. - The fine structure of the spermathecae and their ducts in the mosquito. Aedes aegypti. J. Insect Physiol., 13, 1825-1836.

Eidmann H., 1922. - Die Durchlässigkeit des Chitins bei osmotischen Vorgängen. Biol. Zbl., 42, 429-435.

Flanders S.E., 1946. - Control of sex and sex-limited polymorphism in the hymenoptera. Quart. Rev. Biol., 21, 135-143.

Flanders S.E., 1950. - Control of sex in the Honey Bee. Sci. monthly, 71, 235-240.

Fyg W., 1943. - Experimentelle Untersuchungen aber den Eilegeakt der Bienenkönigin. Mitt. Schweiz. Entomol. Ges., 28, 493-521.

FyG W., 1960. - Utber die Ablagerung von Amyloid im Samenblasenepithel der Bienenkönigin Z. angew. Entomol., 45, 415-420.

LAIDLAW H.H., 1944. - Artificial insemination of queen bees. Morphological basis and results. J. Morph., 74, 429.

LeNSKy Y., SCHINDLER H., 1967. - Motility and reversible inactivation of Honeybee spermatozoa in vivo and in vitro. Ann. Abeille, 10, 5-16.

Lensky Y., Alumoy E., 1969. - Proteins in the spermathecae and haemolymph of the queen bee. Comp. Biochem. Physiol., 1-6.

Rothschild (Lord), 1955. - The spermatozoa of the honeybee. Trans. Roy. Entomol. Soc. London, 107, 289-294.

RutTNER F., 1961. - Die Innervation der Fortpflanzungsorgane der Honigbiene (Apis mellifica L.). Z. Bienenforsch., 5, 253-266.

Snodgrass R.E., 1956. - Anatomy of the Honey Bee. Comstock Publishing Associates, Ithaca, New York. 